\title{
Radiation Pattern of a Microstrip Antenna on a Trapezoidal Substrate
}

\author{
Suchana Mishra ${ }^{1}$, Rabindra Kishore Mishra ${ }^{2}$, Srikanta Patnaik ${ }^{3}$ \\ ${ }^{1}$ Department of Electrical and Electronics Engineering, Dayananda Sagar College of Engineering, \\ Bengaluru 560078, Karnataka, India \\ ${ }^{2}$ Department of Electronics, Berhampur University, Berhampur 760007, Odisha, India \\ ${ }^{3}$ Siksha O Anusandhan University, Bhubaneswar 751030, Odisha, India
}

Received: July 7, 2021. Revised: December 29, 2021. Accepted: January 12, 2022. Published: January 14, 2022.

\begin{abstract}
This paper deals with a rectangular microstrip antenna on a trapezoidal substrate. It finds radiation pattern of the antenna using the concept of fractional cross product. Results show that as the fraction goes from 1 to $\mathbf{0 . 1}$, the direction of null in the $\mathrm{H}$-plane moves from end fire towards broad side. Also, a back-lobe starts to appear in the H-plane.
\end{abstract}

Keywords - Fractional cross product (FCP), Microstrip antenna, Radiation pattern.

\section{INTRODUCTION}

Popularity of microstrip antenna comes from its advantages like low profile, conformability, low cost and different radiating patch shapes as per requirement. The radiating patch can take any possible shape like rectangle, circle, triangle, ellipse and other regular or irregular geometry. Out of which rectangular patches are the most utilized patch geometry. The comparison and analysis of different shape of patch is done by many researchers $[1,2,3]$ in past. Radiation pattern is perhaps the most important characteristics of an antenna. For microstrip antenna, reported pattern analyses consider the plane intersecting the ground and passing through the radiating edge of the patch or to be perpendicular up to the ground plane. It results in an equivalent rectangular slot between the patch and the ground plane. However to the best of our knowledge there is no reported analysis on radiation pattern of this slot is inclined between the patches and the ground plane. In this paper we consider such inclination of analyzing radiation pattern.

Without inclination the normal to the slot is aligned to the length of the patch. With inclination this normal makes an angle. In other words without inclination the normal is parallel to one of the principal axes and with inclination it makes an angle with principal axes. To obtain an expression for radiation pattern, the slot is modelled as an equivalent magnetic current. Fourier transform of the magnetic current density gives the radiation pattern in the far field. The magnetic current density comes from the cross product of electric field in the slot and the unit vector normal to the slot. Both these vectors are orthogonal to each other where the slot is uninclined. So, the magnetic current is orthogonal to both these vectors. Inclination introduces a perturbation to this situation. Therefore, it becomes difficult to obtain an expression for the radiation pattern. To address this issue we propose to use fractional vector calculus as explained later. Particularly we use fractional cross product between two vectors in our derivation.

The basis of antenna theory is maxwell's equation of electromagnetism. In differential form these equations use divergence and curl operations. These operations are based on differential calculus. Fractional differentiation finds application [4, 5, 6] in various branches of science and technology. Therefore, it is natural to extend this to the domain of electromagnetism. Engheta [7] introduced the concept of fractional calculus in the curl operation and proposed a fractional curl operator. It involved Fourier transform of the curl equation and resulting in vector algebraic equations in the Fourier domain. The curl operation becomes a cross product operation in the Fourier domain. Accordingly, concept of fractional cross product, synonymous to fractional curl, was initiated. Later, these theories found applications in reflection of electromagnetic waves from conducting surfaces [8], propagation of electromagnetic wave in chiral medium $[9,10]$.

In this work we use fractional cross product. Application of geometrical perspective [11] eliminates fuzziness associated with fractional cross product discussed earlier.

\section{RADIATION MODEL}

The microstrip antenna has two radiating edges and two nonradiating edges. The radiation model considers space between the ground plane and the radiating edge as a slot. The electric field in this slot is responsible for radiation. A slot is equivalent to a magnetic current ( $M_{g}$ ) obtained from the cross product of normal to the slot ( $\widehat{n})$ with the electric field $(\boldsymbol{E})$ between the radiating edge and the round plane. 


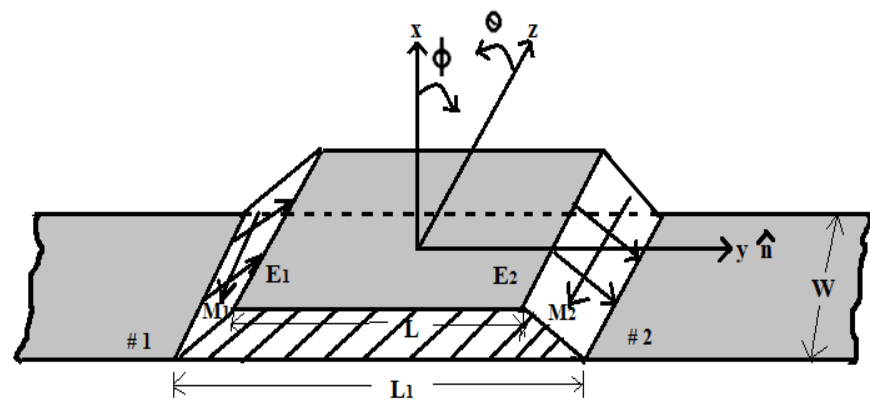

Figure 1. Schematic of a microstrip antenna on a trapezoidal substrate

In this work, the substrate is trapezoidal (figure 1). Therefore, $M_{g}$ is due to a slot which is at an inclination from the ground plane to the patch. $E$ and $M_{s}$ are respectively along $\mathrm{x}$ and $\mathrm{z}$ axes. So, $\hat{n}$ is not orthogonal to the $M_{g}$ as in case of a simple rectangular patch antenna. $M_{g}$ comes from the cross product of $\hat{n}$ and $E$ but not orthogonal to $\hat{n}$. Therefore, concept of integral cross product is insufficient in obtaining $M_{s}$.

Figure 2 helps in explaining the geometrical concept of fractional cross product. It contains two vectors $\boldsymbol{E}$ and $\hat{n}$ representing electric field and the normal to the slot. A projection of $\hat{n}$ to $E$ is $|\hat{n}| \cos \gamma$. Orthogonal to this projection is $\mid \hat{n} \| \sin \gamma$. Direction of the integral cross product $\hat{n} \times \boldsymbol{E}=\|\hat{n}\||\boldsymbol{E}|_{\sin \gamma} \widehat{M}_{g}$ is along $\boldsymbol{E}$ rotated anticlockwise about the axis $|\hat{n}| \sin \gamma$ by $\frac{\pi}{2}$ shown as dashed line in the figure. This line is orthogonal to both $\hat{n}$ and $E$. For fractional cross product the rotation of $E$ about the axis $|\hat{n}| \sin \gamma$ is by an amount $\alpha \frac{\pi}{2}(0 \leq \alpha \leq 1)$ shown by solid line. The symbolic representation of fractional cross product is $(\hat{n} \times)^{\alpha} \boldsymbol{E}$, where $(\hat{n} x)^{\alpha}$ is a linear operator. Table 1 shows the result for fractional cross operation on unit vectors along principal coordinate axes.

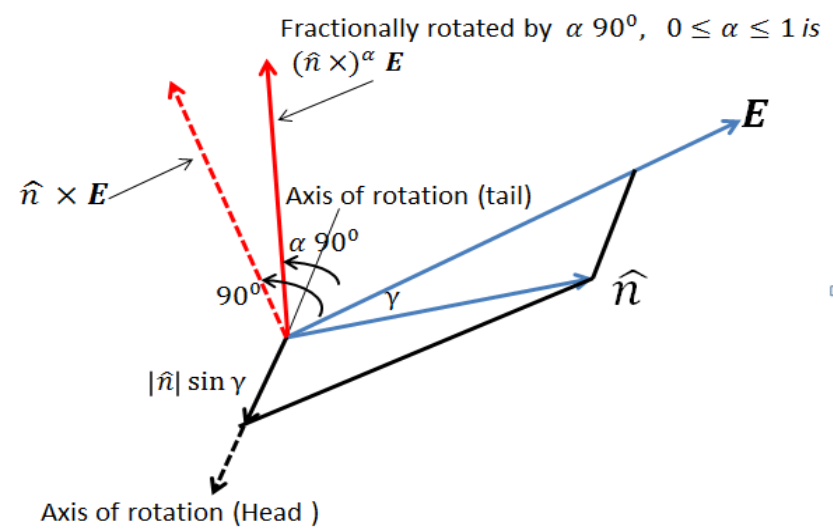

Figure 2. Geometrical concept of fractional cross product

Table 1. Fractional cross products on unit vectors

\begin{tabular}{|l|l|}
\hline$(\hat{z} \times)^{\alpha} \hat{x}$ & $=\left[\cos \left(\frac{\alpha \pi}{2}\right)\right] \hat{x}+\left[\sin \left(\frac{\alpha \pi}{2}\right)\right] \hat{y}$ \\
\hline$(\hat{z} \times)^{\alpha} \hat{y}$ & $=\left[-\sin \left(\frac{\alpha \pi}{2}\right)\right] \hat{x}+\left[\cos \left(\frac{\alpha \pi}{2}\right)\right] \hat{y}$ \\
\hline$(\hat{z} \times)^{\alpha} \hat{z}$ & $=\left[\cos \left(\frac{\alpha \pi}{2}\right)\right] \hat{y}+\left[\sin \left(\frac{\alpha \pi}{2}\right)\right] \hat{z}$ \\
\hline$(\hat{x} \times)^{\alpha} \hat{y}$ & $=\left[-\sin \left(\frac{\alpha \pi}{2}\right)\right] \hat{y}+\left[\cos \left(\frac{\alpha \pi}{2}\right)\right] \hat{z}$ \\
$(\hat{x} \times)^{\alpha} \hat{z}$ & $=\left[\sin \left(\frac{\alpha \pi}{2}\right)\right] \hat{x}+\left[\cos \left(\frac{\alpha \pi}{2}\right)\right] \hat{z}$ \\
\hline$(\hat{y} \times)^{\alpha} \hat{z}$ & $=\left[\cos \left(\frac{\alpha \pi}{2}\right)\right] \hat{x}+\left[-\sin \left(\frac{\alpha \pi}{2}\right)\right] \hat{z}$ \\
\hline$(\hat{y} \times)^{\alpha} \hat{x}$ &
\end{tabular}

\section{RADIATION PATTERN OF THE ANTENNA}

Standard procedure $[12,13]$ gives the radiation pattern in the far field. In this procedure, image theory creates image of $M_{g}$ on the opposite side of the ground plane. This is equivalent to removing the ground plane and doubling $M_{g}$. Corresponding to two radiating edges, there are two magnetic current sources separated by a distance $L_{\text {eff }}$. This distance is due to fringing fields given as

$$
L_{\text {eff }}=L+2 \Delta L
$$

where $\Delta L=0.412 h \frac{\varepsilon_{r v f f}+0.93}{\varepsilon_{r g f f}-0.258}\left(\frac{\frac{W}{h}+0.264}{\frac{W}{h}+0.913}\right)$ is the extended length of the patch.

and $\quad \varepsilon_{\text {reff }}=\frac{\varepsilon_{r}+1}{2}+\frac{\varepsilon_{r}-1}{2}\left(1+\frac{12 h}{W}\right)^{-0.5}$ is the effective dielectric constant.

$\varepsilon_{Y}$ is dielectric constant of substrate, $h$ is the height of dielectric substrate and $W$ is width of the patch of antenna (figure 1).

Thus it is a two element array of magnetic sources. The array factor (AF) of this array is

$$
\mathrm{AF}=2 \cos \left(\frac{K_{0} L_{\text {sff }} \sin \theta \sin \phi}{2}\right)
$$

The element pattern $f(\theta, \phi)$ comes from Fourier transform (FT) of $M_{s}$. Since $M_{s}$ exists only within the slot, so the dimension of the slot determines the limit of integration for finding the FT. For integration it is important to obtain $M_{g}$. As discussed already, for the microstrip antenna on trapezoidal substrate, fractional cross operation on $\mathbf{E}$ gives the $M_{g}$.

$$
M_{g}=-2(\hat{n} \times)^{\alpha} E
$$

As $\hat{n}$ is along y-axis (for normal microstrip antenna) and $\boldsymbol{E}$ $\left(=E_{0} \hat{x}\right)$ is along $\mathrm{x}$-axis, so 


$$
M_{g}=-2\left[(\hat{y} \times)^{\times} \hat{x}\right] E_{0}
$$

From table 1,

$$
(\hat{y} \times)^{\infty} \hat{x}=\left[\cos \left(\frac{\omega \pi}{2}\right)\right] \hat{x}+\left[-\sin \left(\frac{\alpha \pi}{2}\right)\right] \hat{z}
$$

Therefore,

$$
M_{g}=-2 E_{0}\left\{\left[\cos \left(\frac{\alpha \pi}{2}\right)\right] \hat{x}-\left[\sin \left(\frac{\alpha \pi}{2}\right)\right] \hat{z}\right\}
$$

In this expression $M_{g}$ is independent on $\mathrm{x}$ and $\mathrm{y}$. So it can be taken out of integration in the FT and accordingly after conversion to spherical coordinate system patterns for the magnetic current element are,

$$
E_{\theta}=-j \frac{k_{0} e^{-j k_{0} r}}{4 \pi y} N_{\phi}, N_{\phi}=0
$$

So,

$$
E_{\theta}=0
$$

$$
\begin{aligned}
E_{\phi}= & \frac{k_{0} \theta^{-j k_{0} r}}{4 \pi r} N_{\theta}, \\
N_{\theta}= & -2 E_{0} W h \frac{\sin (Z)}{z} \frac{\sin (X)}{x} \\
& {\left[\cos \theta \cos \phi\left[\cos \left(\frac{\alpha \pi}{2}\right)\right]+\sin \theta\left[\sin \left(\frac{\alpha \pi}{2}\right)\right]\right] }
\end{aligned}
$$

$$
\text { So, } \begin{aligned}
E_{\phi}= & -j \frac{k_{0} w \mathrm{~h} E_{0} \mathrm{e}^{-j k_{0} r}}{2 \pi r} \frac{\sin (z)}{z} \frac{\sin (X)}{x} \\
& {\left[\cos \theta \cos \phi\left[\cos \left(\frac{\alpha \pi}{2}\right)\right] \hat{a}_{x}+\sin \theta\left[\sin \left(\frac{\alpha \pi}{2}\right)\right]\right] }
\end{aligned}
$$

Therefore the total pattern for the microstrip antenna is

$$
E_{\phi}^{t}=E_{\phi}(\mathrm{AF})=E_{\phi}\left[2 \cos \left(\frac{k_{0} L_{\text {aff }} \sin \theta \sin \phi}{2}\right)\right]
$$

$E_{\phi}^{t}=-j \frac{k_{0} w V_{0} e^{-j k_{0} r}}{\pi r} \cdot \frac{\sin \left(\frac{k_{n} w}{2} \cos \theta\right)}{\frac{k_{0} W_{w}}{2} \cos \theta} \cdot \frac{\sin \left(\frac{k_{n} h}{2} \cos \phi \sin \theta\right)}{\frac{k_{0} h}{2} \cos \phi \sin \theta}$.

$$
\begin{aligned}
& \cos \left(\frac{\kappa_{0} L_{\text {nff }} \sin \theta \sin \phi}{2}\right) \\
& {\left[\cos \theta \cos \phi\left[\cos \left(\frac{\alpha \pi}{2}\right)\right]+\sin \theta\left[\sin \left(\frac{\alpha \pi}{2}\right)\right]\right]}
\end{aligned}
$$

Where, $\mathrm{h} E_{0}=V_{0}$ is the voltage between the patch edge and the ground plane.

Setting condition for $E$-Plane:

$$
\theta=90^{\circ}, 0^{\circ} \leq \Phi \leq 90^{\circ}, 270^{\circ} \leq \Phi \leq 360^{\circ}
$$

equation (11) gives,

$$
\begin{aligned}
E_{\phi}^{t}(E-\text { plane })= & -j \frac{k_{0} w W_{0} \theta^{-j k_{0} r}}{\pi r} \cdot \frac{\sin \left(\frac{k_{0} h}{2} \cos \phi\right)}{\frac{k_{0} h}{2} \cos \phi} . \\
& \cos \left(\frac{k_{0} L_{\text {sff }} \sin \phi}{2}\right)\left[\sin \left(\frac{\alpha \pi}{2}\right)\right]
\end{aligned}
$$

Setting condition for $\mathrm{H}$-Plane:

$$
\phi=0^{\circ}, 0^{\circ} \leq \theta \leq 180^{\circ}
$$

equation (11) gives,

$$
\begin{gathered}
E_{\phi}^{t}(H-\text { plane })=-j \frac{k_{0} w v_{0} e^{-j k_{0} r}}{\pi r} \cdot \frac{\left.\sin \frac{i k_{n} w}{2} \cos \theta\right)}{\frac{k_{0} w}{2} \cos \theta} \cdot \frac{\sin \left(\frac{k_{0} h}{2} \sin \theta\right)}{\frac{k k_{0} h}{2} \sin \theta} \\
{\left[\cos \theta\left[\cos \left(\frac{\alpha \pi}{2}\right)\right] \hat{a}_{x}+\sin \theta\left[\sin \left(\frac{\alpha \pi}{2}\right)\right]\right]}
\end{gathered}
$$

\section{Results And discussions}

The present study has considered the following values, dielectric constant $\left(\varepsilon_{\gamma}\right)=2.2$, height of the dielectric substrate $(h)=0.1588 \mathrm{~cm}$, position of the recessed feed point relative to the leading radiating edge of the patch $=0.3126 \mathrm{~cm}$, length of the patch $(L)=0.9061 \mathrm{~cm}$, width of the patch $(W)=1.186 \mathrm{~cm}$ and operating frequency $10 \mathrm{GHz}$. For simulation, equations (12) and (13) are implemented in MATLAB. Figure 3 shows the radiation pattern for $\alpha=1$. Under this condition the radiator is a normal rectangular microstrip antenna. Simulation shows that pattern is omni directional in E-plane and figure of 8 in H-plane. In E and $\mathrm{H}$ planes the HPBW (half power band width) are $88^{\circ}$ and $76^{\circ}$ respectively. The directivity of antenna is 5.3506 (i.e. $7.2841 \mathrm{~dB}$ ). Figure 4 shows the pattern for $\alpha=$ 0.95 . The E-plane pattern remains omni while in the H-plane pattern there is a small but prominent back lobe. The E- and H- plane HPBW are $88^{\circ}$ and $82^{\circ}$ respectively. So, decrease of $\alpha$ affects the H-plane HPBW. The directivity of this antenna is 5.3629 (i.e. $7.2940 \mathrm{~dB}$ ). A small incremental change in directivity is evident. Figure 5 shows the pattern for $\alpha=0.8$. The HPBW for E- and H-plane are $80^{\circ}$ and $102^{\circ}$ respectively. So, with appreciable decrease in , HPBW increases for Hplane but decreases in E-plane. However, the overall directivity increases to 5.5527 (i.e.7.4450 dB). Figure 6 shows the pattern when $\alpha=0.1$. For this sufficient decrease in $\alpha, \mathrm{H}$ plane HPBW shows $180^{\circ}$ degree. The peaks of E- and H-plane patterns are in opposite directions. The null in the H-plane pattern is in the direction of E-plane pattern peak. The directivity of the antenna increases sufficiently to 10.4138 (i.e.10.1761dB). 


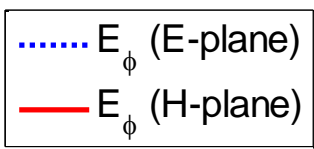

$\ldots . . . E_{\phi}($ E-plane $)$
$-E_{\phi}(H$-plane $)$

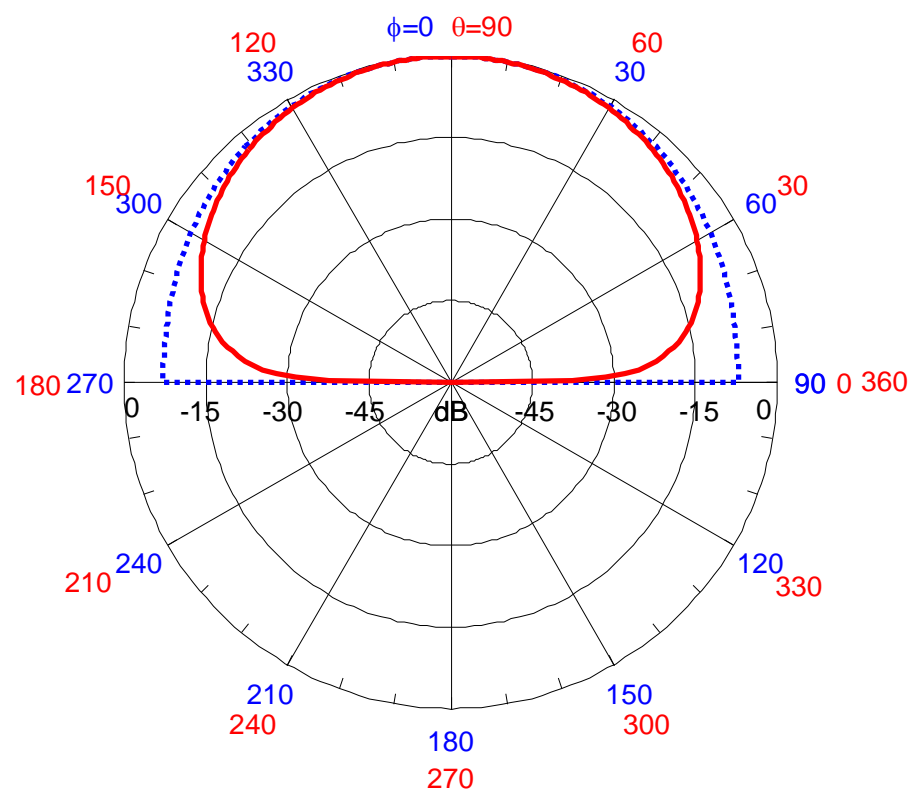

Figure 3. E and $\mathrm{H}$ plane pattern of trapezoidal microstrip antenna for $\alpha=1$

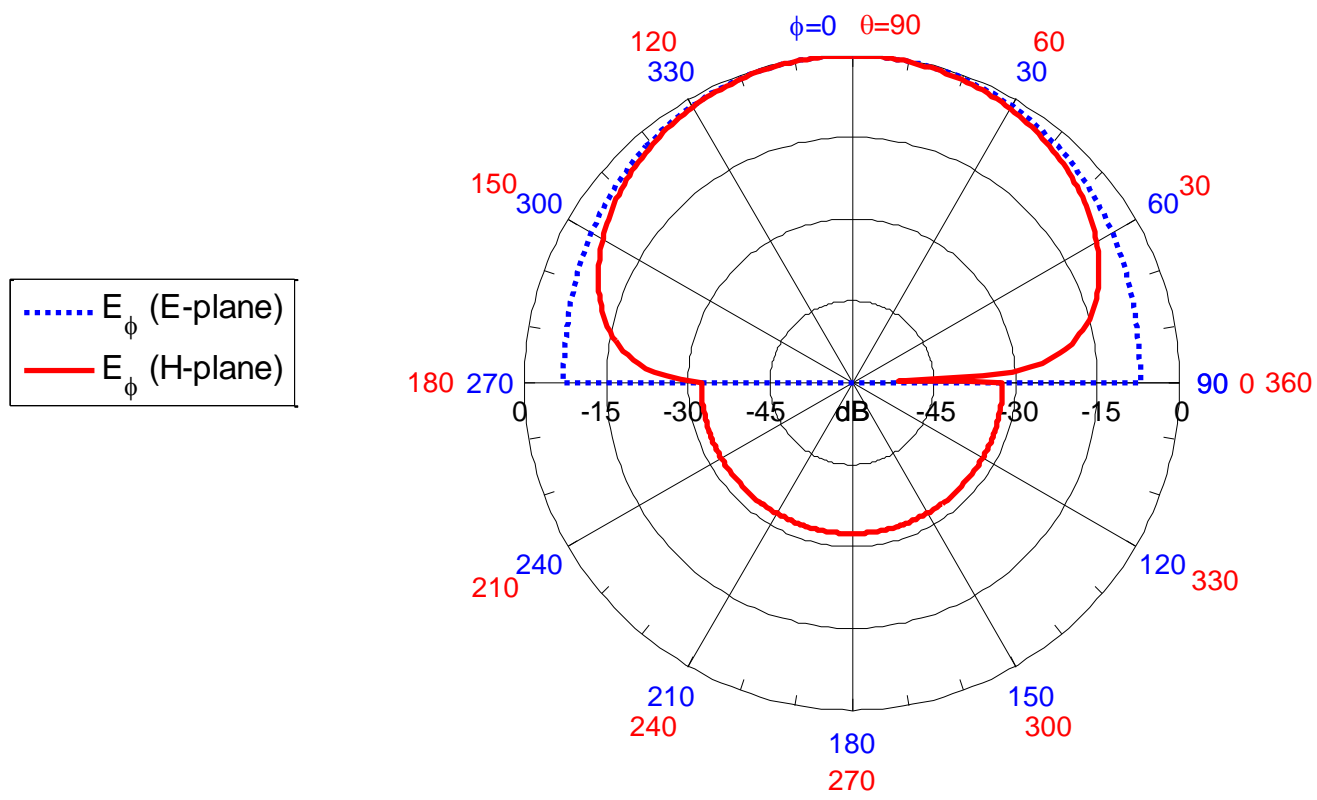

Figure 4. $\mathrm{E}$ and $\mathrm{H}$ plane pattern of trapezoidal microstrip antenna for $\alpha=0.95$ 


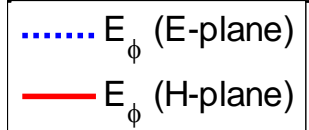

$\ldots \ldots \ldots . . . E_{\phi}($ E-plane $)$
$E_{\phi}(\mathrm{H}$-plane $)$

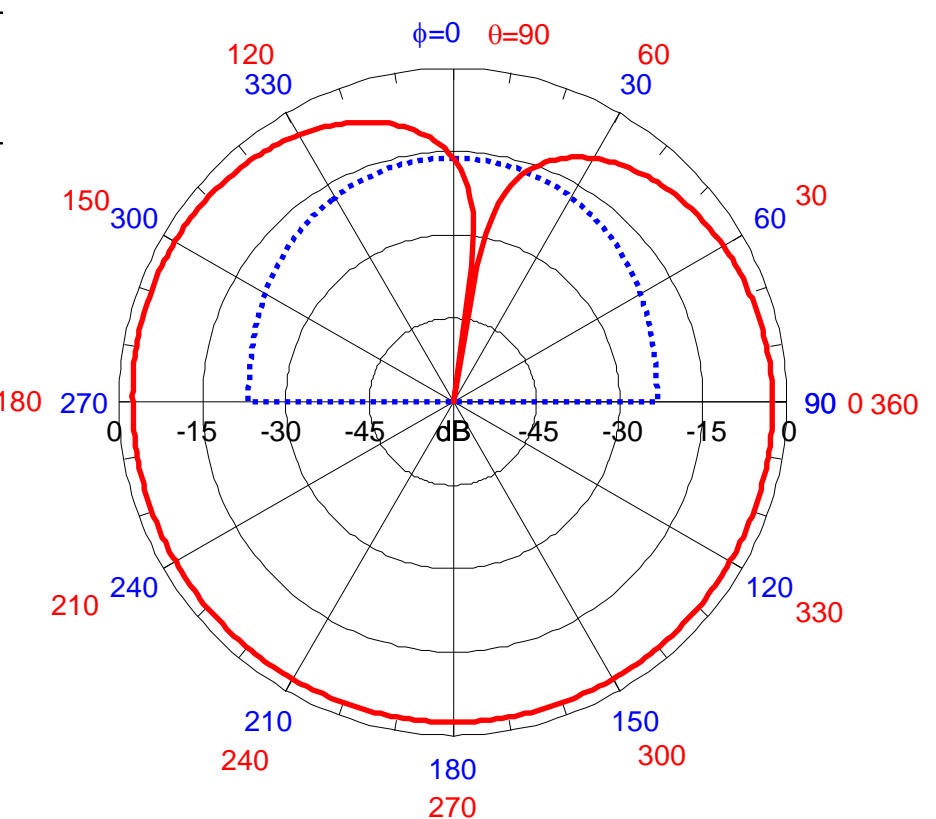

Figure 6. E and $\mathrm{H}$ plane pattern of trapezoidal microstrip antenna for $\alpha=0.1$

\section{Conclusion}

A trapezoidal substrate is more directive compare to that on a brick substrate. The appropriate choice of $\alpha$, the H-plane null can be placed as per requirement. Therefore, a rectangular patch antenna on a trapezoidal surface is advantageous.

\section{References}

[1] M. Meena, P. Kannan,"Analysis of microstrip patch antenna for four different shapes and substrates", ictact journal on microelectronics, ISSN: 2395-1680 (ONLINE), volume: 04, issue: 01 DOI: 10.21917/ijme.2018.0092 519, April 2018.

[2] Eva Karla Garza-Resendiz, Mario Reyes-Ayala, Edgar Alejandro Andrade-Gonzalez, Hilario Terres-Peña, 'USlot Cut Rectangular Microstrip Antenna with a Large Ground Plane and a Thin Substrate", WSEAS Transactions on Communications, Volume 20, 2021, pp. 12-17.

[3] Mohamed F. Abdulmajid, "Study and Analysis of Rectangular Microstrip Patch Antenna at $28 \mathrm{GHz}$ for $5 \mathrm{G}$ 
Applications", WSEAS Transactions on Communications, Volume 20, 2021, pp. 6-11.

[4] Suchana Mishra, Rabindra Kishore Mishra, Srikant Patnaik, "some applications of fractional calculus in technological development", Journal of Fractional Calculus and Application, ISSN: 2090-5858, Volume 10, Issue 1, pp. 228-235, January 2019.

[5] S Das, "Functional Fractional calculus", $2^{\text {nd }}$ edition, Springer- Verlag, Germany, 2011.

[6] S Das, "Functional Fractional Calculus for system identification and controls", Springer Verlag, Germany, 2007.

[7] Engheta N, "Fractional curl operator in electromagnetic", Microwave Opt. Tech. Lett. Vol. 17, 86-91, 1998.

[8] Veliev E.I, Engheta N, “ Fractional curl operator in reflection problems", $10^{\text {th }}$ Int. Conf. on Mathematical Methods in Electromagnetic Theory, 14-17, Ukraine, 2004.

[9] Naqvi S.A, Naqvi Q. A., Hussain A, "Modelling of transmission through a chiral slab using fractional curl operator" Optics Communications, Volme 21, 110-133, 2006.

[10] Hussain A, Naqvi Q. A, "Fractional curl operator in chiral medium and non- symmetric transmission line," Progress in Electromagnetic Research PIER 59, 199213, 2006.

[11] S. Das,"Geometrically deriving fractional cross product and fractional curl", IJMC, Vol.20, Issue 3, 2013.

[12] Balanis C. A., "Antenna Theory: Analysis and Design", 3rd Edition, 3 ed. Wiley-Interscience, Apr. 2005.

[13] Balanis C. A., “Advanced Engineering Electromagnetics", 2nd Edition, John Wiley \& Sons Inc.

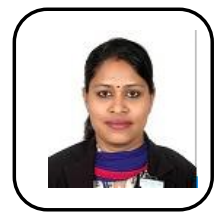

Suchana Mishra is working as assistant professor in Dayananda Sagar College of Engineering, Bangalore, India since 2009. She has done B.Tech and M.Tech from Biju Pattanik University of Technology in the year 2006 and 2008 respectively and pursuing $\mathrm{PhD}$ at SOA University, Bhubaneswar, India. She has supervised more than 15 undergraduate theses and has published around 10 papers in international/national journals, conference proceedings and seminars. She is Life Member of the society, an organisation for promoting the quality and standards in technical education at The Indian Society for Technical Education (ISTE).

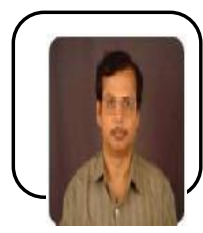

Dr. Rabindra Kishore Mishra is a professor in the Electronic Science Department of the Berhampur University since 2007. He has received his $\mathrm{Ph}$. D. in Electronics from Sambalpur University, India. $\mathrm{He}$ has researched extensively in the areas of planar antennas and applications of soft-computing techniques for analysis and design of planar antennas. He visited the
University of Birmingham as a British Commonwealth Fellow during 1999-2000. He has supervised 14 doctoral theses, published two mono-graphs and over 150 learned articles in journals of repute and pro-ceedings of conferences, seminars, and so on, which earned him IETE Sir J. C. Bose best application paper award (1999) and Shri Hari Ohm Ashram Prerit Hariballabha Das Chunilal Research Endowment Award (2000), Samanta Chandra Sekhar Award in Engineering and Technology (2008), which is the highest award by the Govt. of Orissa. He is also recepient of INSA best teachers award 2015. He had organized short courses on ANN applications for Microwave and Antennas. He is a reviewer for the IEEE Transactions on Antennas and Propagation.

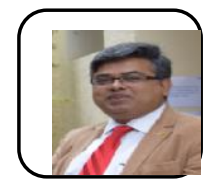

Dr. Srikanta Patnaik is a Professor in the Department of Computer Science and Engineering, Faculty of Engineering and Technology, SOA University, Bhubaneswar, India. He has received his $\mathrm{Ph}$. D. (Engineering) on Computational Intelligence from Jadavpur University, India in 1999 and supervised $25 \mathrm{Ph}$. D. Theses and more than 60 Master theses in the area of Computational Intelligence, Soft Computing Applications and Re-Engineering. Dr. Patnaik has published around 100 research papers in international journals and conference proceedings. He is author of 2 text books and 32 edited volumes and few invited book chapters, published by leading international publisher like Springer-Verlag, Kluwer Academic, etc. Dr. Patnaik is the Editors-in-Chief of International Journal of Information and Communication Technology and International Journal of Computational Vision and Robotics published from Inderscience Publishing House, England and also Editors-in-chief of Book Series on "Modeling and Optimization in Science and Technology" published from Springer, Germany, Book Series on Advances in Computer and Electrical Engineering (ACEE) and Book Series on Advances in Medical Technologiesand Clinical Practices (AMTCP), published from IGI-Global, USA. He is Editor of Journal of Information and Communication Convegence Engineering, published by Korean Institute of Information and Communication Convegence Engineering. $\mathrm{He}$ is also Associate Editor of International Journal of Granular Computing, Rough Sets and Intelligent Systems (IJGCRSIS) and International Journal of Telemedicine and Clinical Practices, published from Inderscience Publishing House, England.

\section{Creative Commons Attribution License 4.0 (Attribution 4.0 International, CC BY 4.0)}

This article is published under the terms of the Creative Commons Attribution License 4.0 https://creativecommons.org/licenses/by/4.0/deed.en US 